Note

\title{
Inhibitory Activity of Hydroxytyrosol against Streptolysin O-Induced Hemolysis
}

\author{
KAZUYUKI SOGAWA ${ }^{1}{ }^{*}$, MIKA KOBAYASHI $^{1}$, JUN SUZUKI ${ }^{1}$, AKIHIRO SANDA ${ }^{1}$, \\ YOSHIO KODERA ${ }^{2}$, AND MASAFUMI FUKUYAMA ${ }^{1}$ \\ ${ }^{1}$ School of Life and Environmental Science, Azabu University, 1-17-71 Fuchinobe, Chuo-ku, Sagamihara, \\ Kanagawa 252-5201, Japan \\ ${ }^{2}$ School of Science, Kitasato University, 1-15-1 kitasato, Minami-ku, Sagamihara, Kanagawa 252-0373, Japan \\ Received 16 February, 2016/Accepted 2 March, 2017

\begin{abstract}
Group A streptococcus is a bacterium that resides in the throat and skin and causes respiratory infection and occasionally glomerulonephritis and rheumatic fever. Streptolysin 0 (SLO) produced by Streptococcus pyogenes (S. pyogenes) binds to the cell membrane, particularly to that of white and red blood cells, and is toxic to the cells and tissue. In this study, we evaluated the inhibitory activity of water-soluble polyphenols in olives (Olea europaea) against SLO-induced hemolysis. Hydroxytyrosol inhibited SLO-induced hemolytic activity, and the amount required for $50 \%$ inhibition of hemolysis was $1.30 \mu \mathrm{g}$. These findings suggest that the water-soluble polyphenols contained in olives have inhibitory activity against SLO-induced hemolysis.
\end{abstract}

Key words : Streptococcus pyogenes / Olea europaea / Streptolysin O / Hydroxytyrosol.

Group A streptococcus (GAS) is a bacterium that resides in the throat and skin and causes respiratory infection and occasionally glomerulonephritis and rheumatic fever. GAS evokes streptococcal toxic shock-like syndrome (STSS), accompanied by critical illnesses such as soft tissue necrosis and multiple organ failure (Shiseki et al., 1999). Therefore, reemerging infectious diseases caused by GAS are of renewed interest, and the food-borne epidemics due to GAS have emerged as a social issue. Such outbreaks have been reported in Israel, Europe, the United States, and China from 1992 to 2006 (Asteberg et al., 2006; Kaluski et al., 2006; Pournaras et al., 2000; Yang et al., 2007).

Since the late 1990s, epidemiological analyses of seven food-borne outbreaks have been reported in Japan (Tanaka et al., 2006). However, the relationship between virulence factors and pathosis has not been examined in food-borne isolates. In July 2005, a large food poisoning incident caused by GAS (Streptococcus pyogenes type T-25) was reported in Kanagawa Prefecture involving 489 people, of which 218 cases were associated with an open university campus (Suzuki

*Corresponding author. Tel·Fax: +81-42-769-1924, E-mail : sogawa (a)azabu-u.ac.jp et al., 2006). We analyzed the activity of three major virulence factors produced by this strain: streptolysin $\mathrm{O}$ (SLO), cysteine protease streptococcal pyrogenic exotoxin B (SpeB), and NAD glycohydrolase (NADase) (Kanno et al., 2012).

The major polyphenols in olives are hydroxytyrosol and acteoside (Bullon et al., 2009). Hydroxytyrosol is found in the fruit and in olive oil, and has been shown to have preventive effects on arteriosclerosis due to its antioxidative activity in an animal model (Bullon et al., 2009). Hydroxytyrosol included in the olive oil ranges from 1.34 to $3.30 \mathrm{mg} / \mathrm{kg}$, expressed as the 10 th and 90th percentiles of the data distribution, respectively (Gomez-Rico et al., 2006). Streptolysin O (SLO) produced by $S$. pyogenes binds to the cell membrane, particularly that of white and red blood cells, and is toxic to cells and tissue (Suzuki, 2009). In this study, we modified the microplate method of Pinkney et al. (1989) for evaluation of hemolysis. We used this method to evaluate the inhibitory activity of water-soluble polyphenols in olives (Olea europaea) against SLO-induced hemolysis.

Ripe and immature olives (variety: Mission, Yamada Olive Garden) were weighed (20 g each), crushed, and filtered through gauze, followed by centrifugation 


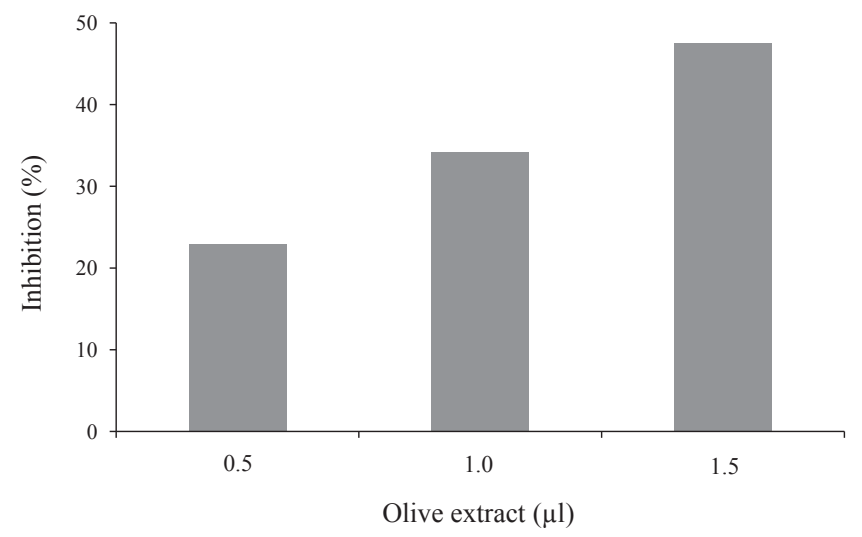

FIG. 1. Rates of inhibition of SLO-induced hemolysis with immature olive extracts

$(21,130 \times \mathrm{g}, 15 \mathrm{~min})$, and $2 \mathrm{ml}$ of the water-soluble components were collected. The samples were stored at $-80^{\circ} \mathrm{C}$.

Hemolytic activity was spectrophotometrically determined using a 50\% endpoint titration (Kusama et al., 1958). With reference to this method of Kusama et al. (1958), we established a microplate method with the amounts of reagents and sample reduced to $1 / 10$ the scale of the original method. Pure SLO (Wako Pure Chemical Industries, Ltd., Osaka, Japan) was diluted on a 0.1 log-unit dilution scale with phosphate-buffered saline (PBS, pH 6.5) supplemented with 2-mercaptoethanol (2-ME) and bovine serum albumin. Test samples (olives, hydroxytyrosol (Wako Pure Chemical Industries)) were added in the microplate (Sumitomo Bakelite Co. Ltd, Tokyo, Japan), and then $50 \mu$ of a $2.5 \%$-suspension of rabbit erythrocytes (Nippon Bio-Supp. Center, Tokyo, Japan) in PBS was added to $150 \mu$ of solution. After incubation at $37^{\circ} \mathrm{C}$ for $1 \mathrm{~h}$, tubes were centrifuged, and absorbance of the supernatant was measured at $510 \mathrm{~nm}$ against a blank containing the diluent.

Activity was expressed as the $50 \%$ hemolytic dose $\left(H D_{50}\right)$ per ml. To determine hemolytic efficiency (HE), the $\%$ hemolysis was calculated for each tube and converted into a probit. A rectilinear curve was obtained by plotting the probit against the log of the hemolysin dose. HE was obtained from this curve using the formula: $\mathrm{HE}=\left[N \mathrm{NP}_{i} y_{i}-\mathrm{P} x_{i} \mathrm{P} y_{i}\right] /\left[N P x_{i}^{2}-\left(P x_{i}\right)^{2}\right]$, where $x$ is the log of the hemolysin dose, $y$ is the \% lysis in the probits, and $\mathrm{N}$ is the number of points in the rectilinear curve.

The hemolytic potencies were $1.51 \times 10^{3} \pm 96.2 \mathrm{HD}_{50} \mathrm{l}$ $\mathrm{ml}(\mathrm{CV} 6.8 \%), 1.22 \times 10^{3} \pm 124.3 \mathrm{HD}_{50} / \mathrm{ml}$ (CV $15.2 \%$ ), and $1.01 \times 10^{3} \pm 102.9 \mathrm{HD}_{50} / \mathrm{ml}$ (CV 10.2\%) after addition of $0.5,1.0$, and $1.5 \mu \mathrm{l}$ of immature olive extract, respectively. The inhibition rates were $22.9 \%$,

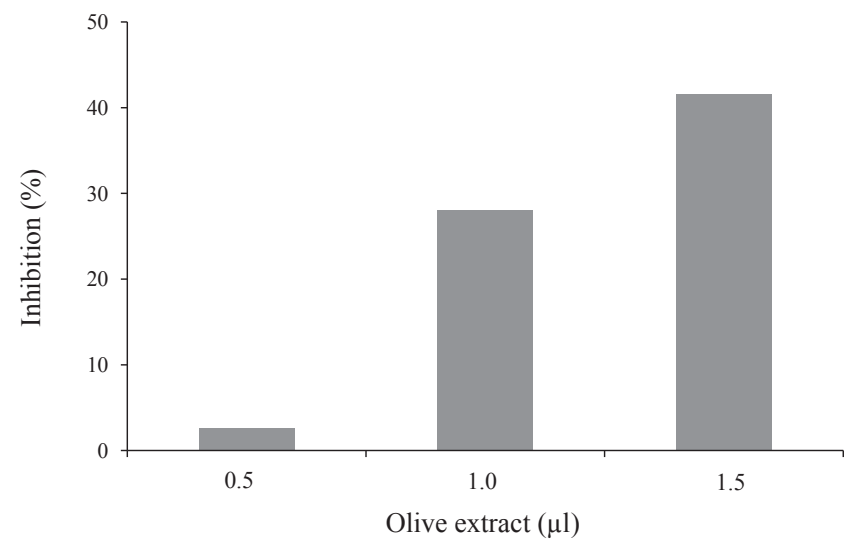

FIG. 2. Rates of inhibition of SLO-induced hemolysis with ripe olive extracts

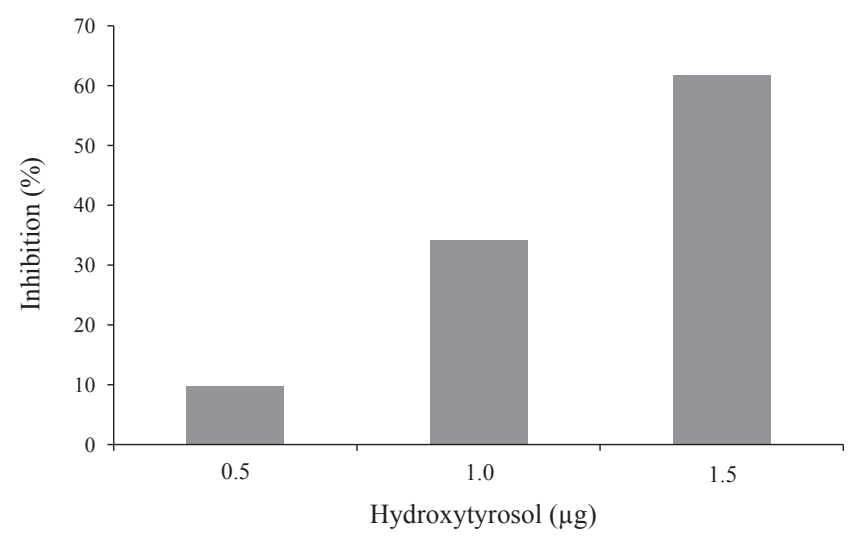

FIG. 3. Rates of inhibition of SLO-induced hemolysis with hydroxytyrosol

$34.2 \%$, and $47.5 \%$, respectively (Fig. 1 ), and $1.47 \mu \mathrm{l}$ of extract was required for $50 \%$ inhibition of hemolysis. The hemolytic potencies were $2.12 \times 10^{3} \pm 265.7 \mathrm{HD}_{50} /$ $\mathrm{ml}(\mathrm{CV} 12.8 \%), 1.56 \times 10^{3} \pm 114.2 \mathrm{HD}_{50} / \mathrm{ml}(\mathrm{CV}$ $26.2 \%$ ), and $1.26 \times 10^{3} \pm 95.2 \mathrm{HD}_{50} / \mathrm{ml}$ (CV 8.1\%) after addition of $0.5,1.0$, and $1.5 \mu \mathrm{l}$ of ripe olive extract, respectively. The inhibition rates were $2.6 \%, 28.0 \%$, and $41.6 \%$, respectively (Fig.2), and $1.78 \mu$ of extract was required for $50 \%$ inhibition of hemolysis. The hemolytic potencies were $1.96 \times 10^{3} \pm 108.1 \mathrm{HD}_{50} / \mathrm{ml}, 1.41 \times 10^{3}$ $\pm 98.4 \mathrm{HD}_{50} / \mathrm{ml}$, and $8.14 \times 10^{3} \pm 42.8 \mathrm{HD}_{50} / \mathrm{ml}$ after addition of $0.5,1.0$, and $1.5 \mu \mathrm{g}$ of hydroxytyrosol, respectively, and the inhibition rates were 9.8\%, 34.2\%, and $61.8 \%$, respectively (Fig.3). The amount required for $50 \%$ inhibition of hemolysis was $1.30 \mu \mathrm{g}$.

Hydroxytyrosol inhibited SLO-induced hemolytic activity, with $1.30 \mu \mathrm{g}$ required for $50 \%$ inhibition of hemolysis. These findings suggest that water-soluble polyphenols contained in olives have inhibitory activity. Olive extracts also inhibited the hemolytic activity of SLO, with $1.47 \mu \mathrm{l}$ and $1.78 \mu \mathrm{l}$ of immature and ripe fruit 
extracts required for $50 \%$ inhibition of hemolysis, respectively. The greater inhibition by immature fruit extract is consistent with the respective polyphenol contents of 0.61 and $0.36 \mathrm{mg} / \mathrm{g}$ in olive oil prepared from immature and ripe fruits of the same variety (Mission) (Shibasaki, 2000). The water-soluble components of immature fruit contain abundant polyphenols, as well as oil components, and this may increase the inhibitory activity. We measured the concentration of hydroxytyrosol using HPLC with Shiseido Co., Ltd. The concentration of hydroxytyrosol was $0.44 \pm 0.03 \mathrm{mg} / \mathrm{g}$ in immature olives and $0.32 \pm 0.02 \mathrm{mg} / \mathrm{mL}$ in ripe olives.

The antitoxic action of tea catechins (epicatechin, epigallocatechin, epicatechin gallate, and epigallocatechin gallate) requires the presence of catechol, pyrogallol, and galloyl groups, and is dependent on the configurations of these groups (Toda et al., 1990). A hydroxytyrosol contained in the water-soluble component of the olive fruit may also influence the configuration of SLO. Hydroxytyrosol $\left(\mathrm{C}_{8} \mathrm{H}_{10} \mathrm{O}_{3}\right)$ possesses only one catechol group.

S. pyogenes infection results in general symptoms of respiratory diseases caused by airborne droplet infection and food poisoning, and may also lead to the development of serious symptoms of fulminant hemolytic streptococcal infection. This infectious disease is frequently fatal, with 57 fatal cases among a total of 143 patients in Japan in 2012 (Streptococcal infections in Japan, April 2006-2011. IASR., 33, 209-210, 2012). In the case of the sudden death of a mother and fetus, high SLO production induced severe hemolytic anemia and pulmonary hemorrhage, and was closely involved in fetal cardiac arrest (Kanno et al., 2011).

Hemolysin-producing bacteria other than S. pyogenes include Staphylococcus aureus, Vibrio parahaemolyticus, Vibro cholera, and Clostridium perfringens, and olives are also likely to have hemolysis inhibitory activity against these bacteria (Toda et al., 1990). No inhibitory effect of tea catechins on the hemolytic activity of the a toxin of Staphylococcus aureus, heat-resistant hemolysin Vp-TDH of Vibrio parahaemolyticus, or cholera toxin was observed, and the effect was similar to that of the negative control. However, hemolytic activity was noted for catechins and their structural analogs, suggesting that the different result was due to a structural difference (Toda et al., 1990). We are planning to investigate the action of water-soluble polyphenols of olives against the hemolysin produced by other bacteria.

In the current study, immature and ripe olive extracts showed inhibitory activity against SLO-mediated hemolysis produced by S. pyogenes. Hydroxytyrosol, which is a water-soluble polyphenol found abundantly in olives, also showed hemolysis inhibition.

\section{ACKNOWLEDGMENTS}

This study was partially supported by a research grant awarded by Azabu University.

\section{REFERENCES}

Alouf, J. E. (1980) Streptococcal toxins (streptolysin O, streptolysin S, erythrogenic toxin). Pharmacol. Ther., 11, 661717.

Anonymous. (2012) Streptococcal infections in Japan, April 2006-2011. IASR., 33, 209-210.

Asteberg, I., Andersson, Y., Dotevall, L., Ericsson, M., Darenberg, J., Henriques-Nordmark, B., and Söderström, A. (2006) A food-borne streptococcal sore throat outbreak in a small community. Scand. J. Infect. Dis., 38, 988-994.

Bordiga M, Lorenzo C, Pardo F, Salinas MR, Travaglia F, Arlorio M, Coïsson JD, Garde-Cerdán T. Factors influencing the formation of histaminol, hydroxytyrosol, tyrosol, and tryptophol in wine: Temperature, alcoholic degree, and amino acids concentration.

Bullon, P., Quiles, J. L., Morillo, J. M., Rubini, C., Goteri, G., Granados-Principal, S., Battino, M., and Ramirez-Tortosa M. (2009) Gingival vascular damage in atherosclerotic rabbits: hydroxytyrosol and squalene benefits. Food. Chem. Toxicol., 47, 2327-2331.

Gomez-Rico, A., Salvador, M.D., La Greca, M., and Fregapane, G. (2006) Phenolic and Volatile Compounds of Extra Virgin Olive Oil (Olea europaea L. Cv. Cornicabra) with Regard to Fruit Ripening and Irrigation Management. J. Agric. Food. Chem., 54, 7130-7136.

Kaluski, D. N., Barak, E., Kaufman, Z., Valinsky, L., Marva, E., Korenman, Z., Gorodnitzki, Z., Yishai, R., Koltai, D., Leventhal, A., Levine, S., Havkin, O., and Green, M. S. (2006) A large food-borne outbreak of group A streptococcal pharyngitis in an industrial plant potential for deliberate contamination. Isr. Med. Assoc. J., 8, 618-621.

Kanno, T., Sakaguchi, K., Fukuyama, M., and Suzuki, J. (2011) Properties of metabolic substances produced by group A streptococcus from a food-borne epidemic. J. Infect. Chemother., 17, 462-467.

Kanno, T., Sakaguchi, K., and Suzuki, J. (2012) Time course of virulence factors produced by group A streptococcus during a food-borne epidemic. J. Infect. Chemother., 18, 35-40.

Pinkney, M., Beachey, E., and Kehoe, M. (1989) The thiolactivated toxin streptolysin $\mathrm{O}$ does not require a thiol group for cytolytic activity. Infect Immun., 57, 2553-2558.

Pournaras, S., Efstratiou, A., Douboyas, J., George, R. C., and Tsakris, A. (2000) Genetic relatedness of group A streptococci of the newly designated serotype M90 causing a food-borne outbreak and sporadic infections. Epidemiol. Infect., 125, 299-301.

Shibasaki, H. (2000) Comparison of quality in olive oil from some cultivars (in Japanese). Report of the Food Research Institute and the Fermentation \& Food Experimental Station, Kagawa Prefectural Government., 92, 27-30.

Shiseki, M., Miwa, K., Nemoto, Y., Kato, H., Suzuki, J., Sekiya, K., Murai, T., Kikuchi, T., Yamashita, N., Totsuka, K., Ooe, K., Shimizu, Y., and Uchiyama, T. (1999) Comparison of pathogenic factors expressed by group A streptococci isolated from patients with streptococcal toxic shock syndrome and scarlet fever. Microb. Pathog., 27, 243-252.

Suzuki, J. (2009) Characterization of acidic and neutral strep- 
tolysin O. J. Electrophoresis., 53, 45-50.

Suzuki, R., and Nikkawa, T. (2006) A food-borne epidemic of group A streptococcal pharyngitis (in Japanese). Bull. Kanagawa. Inst. PH., 36, 12-13.

Tanaka, D., Shima, T., Isobe, J., Watahiki, M., Matsumoto, M., Endoh, M., Okuno, R., Ogata, K., and Nagai, Y. (2006) Epidemiology and molecular analysis of group A streptococci from patients involved in foodborne disease outbreaks in Japan between 1996 and 2003 (in Japanese). Jpn. J. Infect. Dis., 59, 202-203.

Toda, M., Okubo, S., Ikigai, H., and Shimamura, T. (1990)
Antibacterial and anti-hemolysin activities of tea catechins and their structural relatives (in Japanese). Nihon. Saikingaku. Zasshi., 45, 561-566.

Visioli, F., Bellomo, G., and Galli, C. (1998) Free radicalscavenging properties of olive oil polyphenols. Biochemical Biophys. Res. Commun., 247, 60-64.

Yang, S. G., Dong, H. J., Li, F. R., Xie, S. Y., Cao, H. C., Xia, S. C., Yu, Z., and Li, L. J. (2007) Report and analysis of a scarlet fever outbreak among adults through food-borne transmission in China. J. Infect., 55, 419-424. 